\title{
Thermal coherence properties of topological insulator slabs in time-reversal symmetry breaking fields
}

\author{
Xiao Xiao, ${ }^{1}$ S. Li, ${ }^{1}$ K. T. Law, ${ }^{1}$ Bo Hou, ${ }^{2}$ C. T. Chan,,${ }^{1}$ and Weijia Wen ${ }^{1, *}$ \\ ${ }^{1}$ Department of Physics, The Hong Kong University of Science and Technology, Clear Water Bay, Kowloon, Hong Kong \\ ${ }^{2}$ School of Physical Science and Technology, Soochow University, No. 1 Shizi Street, Suzhou, Jiangsu 215006, China
}

(Received 25 June 2012; revised manuscript received 9 April 2013; published 20 May 2013)

\begin{abstract}
We first develop a general Green's-function method for the slab geometry, which can handle quite complex conversions between the different polarized radiation modes. Then by using the method, we study the near field thermal coherence properties of a slab of $Z_{2}$ topological insulator (TI) with a finite thickness. Under a strong enough external magnetic field, the gapless helical Dirac fermions on the TI surfaces can acquire an energy gap larger than the photon energy of the radiation field at the Dirac point. This gapped surface states can couple strongly with the waveguide modes in the bulk of the TI slab, which thus induces an electromagnetic resonance. Exactly due to the resonance, the coherence properties of the thermal radiation field can be modified dramatically. For $Z_{2}$ topological insulators, the parameter regime with the surface Hall conductivity half integer quantized is unique. We demonstrated that within the parameter regime, for a TI slab of a proper thickness, the thermal radiation energy density can be enhanced very much, and the coherence length of the thermal radiation field can be as long as twice that of $\mathrm{SiC}$.
\end{abstract}

DOI: 10.1103/PhysRevB.87.205424

PACS number(s): 42.25.Kb, 05.40.-a, 73.20.At

\section{INTRODUCTION}

It is well known that the near field thermal radiation is quite different from the thermal energy transfer in the far field. ${ }^{1-7}$ These differences can be viewed as a consequence of the improved coherence properties ${ }^{8}$ of the radiative field in the near field. Due to its various potential applications, including imaging, lighting, and heat-to-electrical conversion, ${ }^{9-11}$ the manipulation of thermal radiation field has attracted much attention in recent studies. As it has been shown, the coherence properties of the thermal radiation in artificial structures such as microcavities ${ }^{12}$ and photonic crystals ${ }^{13-15}$ can be quite different from that of a black body. In particular, for systems supporting the electromagnetic surface modes, such as the noble metals and $\mathrm{SiC},{ }^{1,2}$ long-range spatial coherence of thermal radiation field can be established, and the improved spatial coherence can cause directional thermal emission ${ }^{3}$ and enhance radiative heat transfer. ${ }^{16-18}$

On the other hand, topological insulators ${ }^{19-22}$ are bulk insulators which have gapless topologically protected surface states. However, in the presence of a time-reversal breaking (TRB) perturbation the surface state of a $Z_{2}$ topological insulator (TI) acquires an energy gap. This gapped surface state can lead to the half integer quantized surface Hall conductivity in response to an electromagnetic field of frequency $\omega,{ }^{23,24}$ when $\hbar \omega$ is much smaller than the surface gap $\Delta$. The gapped surface state is the origin of many interesting effects proposed recently, such as the possibility of inducing magnetic monopoles, ${ }^{25}$ the tunable Casimir effect, ${ }^{26}$ and the giant magneto-optical Kerr effect. ${ }^{27}$

In this paper, we first develop a general Green's-function method for the slab geometry regardless of how complex the scattering processes are. Then, by using this method, we study the effects of the surface states on the coherence properties of thermal radiation field from a $Z_{2}$ TI slab. The schematic configuration of the system under consideration is shown in Fig. 1(a). As the external magnetic field $(B)$ is small, the surface gap $\Delta(\Delta \propto B)$ of the surface states is smaller than the photon energy of the radiation field $(\hbar \omega)$. Thus, the electrons in the lower surface band can be excited to the upper one by thermal radiation, which results in the nonvanishing dissipative surface conductivities $\sigma_{x x}^{R}$ (real part of surface longitudinal conductivity) and $\sigma_{x y}^{I}$ (imaginary part of surface Hall conductivity). In this interband transition regime, the coupling between the surface states and the waveguide modes in the bulk of the TI slab is weak, so the spatial coherence of thermal radiation field does not deviate much from that of a dielectric slab. However, with the increasing external magnetic field, the TI surface states become gapped $(2 \Delta>\hbar \omega)$, which causes the vanishing of the dissipative surface conductivities $\sigma_{x x}^{R}$ and $\sigma_{x y}^{I}$. Under the situation, the coupling between the surface states and the waveguide modes can induce a novel resonance, which indicates a much stronger coupling between them. Importantly, due to the resonance, the spatial coherence of thermal radiation field is dramatically modified from that of a dielectric slab. Moreover, the thermal radiation energy density is found to be enhanced significantly. These illustrate that when the gapped surface states couple strongly with the waveguide modes, more random incoherent thermal energy can be converted into coherent radiation energy. As a specified example, we demonstrated that for a TI slab of a proper thickness both the coherence length and the thermal radiation energy density are dramatically enhanced, when the surface Hall conductivity is half integer quantized. Remarkably, the coherence length of the $x$ component of the radiation field is found to be as long as twice that for $\mathrm{SiC}$.

The paper is organized as follows. In Sec. II, we describe the model of the surface states of a $Z_{2}$ topological insulator and briefly outline the Green's-function formulism to describe the electromagnetic scattering in a slab including the complex mode conversions at the interfaces. In Sec. III, we study the coherence properties of TI slabs and demonstrate the great enhancement of thermal coherence properties due to the gapped surface states. In Sec. IV, we compare our results 

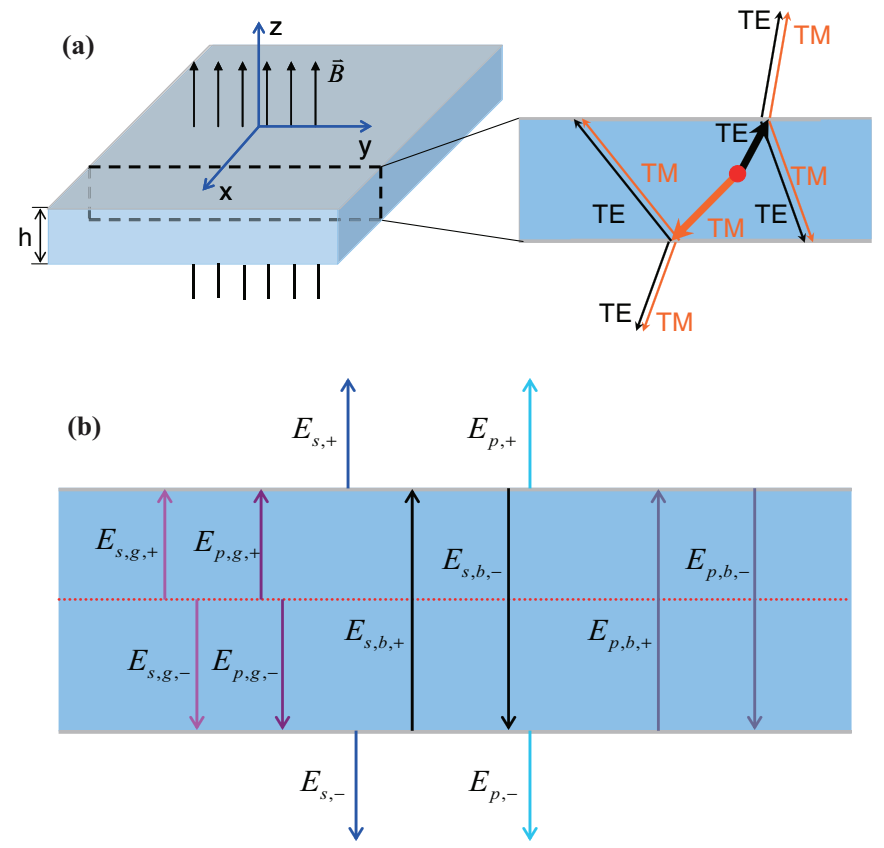

FIG. 1. (Color online) (a) Left panel: The schematic configuration of a TI slab under a magnetic field with the associated coordinate: the origin of the coordinate is on the upper TI/vacuum interface. Right panel: Scattering of thermal radiation fields at the TI surfaces. The black (orange) thick arrow denotes the TE (TM) thermal radiation modes generated from a random current source (the red dot in figure) in a TI slab, and the black (orange) thin arrows denote the scattered TE (TM) modes. (b) The mode expansion scheme for solving the scattering problem. The red dot line indicates the source plane, the pink (violet) arrows indicate the TE (TM) modes generated by the source plane, the black (gray) arrows denote the TE (TM) modes in the bulk of the TI slab, and the blue (sky-blue) arrow presents the TE (TM) modes in vacuum. The source plane is obtained from random current sources by Fourier transformation (for details see the text or Ref. 28).

with those of the system supporting surface modes (i.e., SiC). Finally, the conclusion remarks are provided in Sec. V.

\section{MODEL AND METHODOLOGY}

In this section, we will first present the model of the surface states of a topological insulator. Then we will discuss the cross-spectral density tensor, which directly relates to the coherence properties of the radiation field. The mode conversion between the transversal electric (TE) mode (with the electric field parallel to the TI surfaces) and the transversal magnetic modes (with the magnetic field parallel to the TI surfaces) on the TI surfaces makes the scattering of radiation fields in the system very complex. To solve the problem, we extended the Green's-function method ${ }^{28}$ by using the mode expansion scheme so that we obtained a quite general expression of the Green's function for the slab geometry regardless of how complex the scattering processes are.

\section{A. Model of the surface states of a topological insulator}

The effective Hamiltonian describing the surface states of a $Z_{2}$ topological insulator under a time-reversal breaking external field can be given by ${ }^{29,30}$

$$
\mathcal{H}=v_{F}\left(k_{x} \sigma_{y}-k_{y} \sigma_{x}\right)+\Delta \sigma_{z},
$$

where the first term describes the gapless surface states, and $\Delta$ is the surface gap due to the external field and relates to the external field as $\Delta=g_{s} \mu_{B} B / 2$. In the above, $v_{F}$ is the Fermi velocity, $k_{x}$ and $k_{y}$ are in-plane momentum components of surface electrons, $\sigma_{x}, \sigma_{y}$, and $\sigma_{z}$ are Pauli matrices, $g_{s}$ is the Lande factor of the Dirac fermions, and $\mu_{B}$ is the Bohr magneton.

According to the effective Hamiltonian, the energy spectra is given by $E_{ \pm}(\vec{k})= \pm \sqrt{v_{F}^{2}\left(k_{x}^{2}+k_{y}^{2}\right)+\Delta^{2}}$, which indicates that the external magnetic field opens an energy gap $\Delta=$ $g_{s} \mu_{B} B / 2$ at the Dirac point. The corresponding eigenstates are given by

$|+, \vec{k}\rangle=\left(\begin{array}{c}\cos \frac{\beta}{2} \\ \sin \frac{\beta}{2} \exp \left(i \theta_{\vec{k}}\right)\end{array}\right), \quad|-, \vec{k}\rangle=\left(\begin{array}{c}\sin \frac{\beta}{2} \\ -\cos \frac{\beta}{2} \exp \left(i \theta_{\vec{k}}\right)\end{array}\right)$,

where $\beta$ is defined as $\cos \beta=\frac{\Delta}{\sqrt{v_{F}^{2}\left(k_{x}^{2}+k_{y}^{2}\right)+\Delta^{2}}}$, and $\tan \theta=$ $-k_{x} / k_{y}$.

To describe the response of the surface states to the external electromagnetic field, we use the Kubo's formula to calculate the optical conductivity: ${ }^{31,32}$

$$
\begin{aligned}
\sigma_{\alpha \beta}(\vec{q}, \omega)= & \frac{i e^{2} v_{F}^{2}}{4 \pi^{2}} \sum_{\vec{k}, \lambda, \lambda^{\prime}}\left(\frac{\partial \mathcal{H}}{\partial k_{\alpha}}\right)_{\lambda, \lambda^{\prime}}\left(\frac{\partial \mathcal{H}}{\partial k_{\beta}}\right)_{\lambda^{\prime}, \lambda} \\
& \times \frac{n_{F}\left(\epsilon_{\vec{k}, \lambda^{\prime}}\right)-n_{F}\left(\epsilon_{\vec{k}-\vec{q}, \lambda}\right)}{\left(\omega+\epsilon_{\vec{k}, \lambda^{\prime}}-\epsilon_{\vec{k}-\vec{q}, \lambda}\right)\left(\epsilon_{\vec{k}, \lambda^{\prime}}-\epsilon_{\vec{k}-\vec{q}, \lambda}\right)},
\end{aligned}
$$

where $\lambda$ and $\lambda^{\prime}$ denote the band index, $n_{F}$ is the Dirac-Fermi distribution function, and $\left(\frac{\partial \mathcal{H}}{\partial k_{\alpha}}\right)_{\lambda, \lambda^{\prime}}$ is the expectation value of the $\alpha$-component velocity operator.

After the straightforward evaluation of (3), the longitudinal and Hall conductivity in the unit of $e^{2} / \hbar$ are given by

$\sigma_{x x}^{R}=\left[1 / 16+\Delta^{2} /\left(4 \omega^{2}\right)\right] \Theta[\omega-2 \max (\Delta, \mu)]$,

$\sigma_{x x}^{I}=1 /(2 \pi \omega)\left\{n_{F}(\Delta) \Delta+k_{B} T \ln \left[F(\Delta)^{2}+1\right]\right\}$

$+1 /(8 \pi)\left\{2 \frac{\Delta^{2}}{\omega} \frac{1}{\max (\Delta, \mu)}+\left[1+4(\Delta / \omega)^{2}\right] f(\omega) / 2\right\}$,

$\sigma_{x y}=i \Delta /(4 \omega) \Theta[\omega-2 \max (\Delta, \mu)]-\Delta /(4 \pi \omega) f(\omega)$,

where $f(\omega)=-\ln \left[\frac{|\omega+2 \max (\Delta, \mu)|}{|\omega-2 \max (\Delta, \mu)|}\right], \quad F(\Delta)=\exp [-(\Delta-\mu) /$ $\left.\left(2 k_{B} T\right)\right], n_{F}(\Delta)=\frac{1}{1+\exp \left[(\Delta-\mu) /\left(k_{B} T\right)\right]}, \Theta[\omega-2 \max (\Delta, \mu)]$ is the step function, and $\mu$ is the chemical potential relative to the Dirac point. The $\omega=0$ and $\Delta=0$ limits of our results in (4) agree with the previous results of the Dirac model. ${ }^{27,33,34}$

\section{B. Extended Green's-function method and the cross-spectral density tensor}

The coherence properties of the thermal radiation field is characterized by the cross-spectral density tensor, which is 
the ensemble average of the radiation fields at two different locations: ${ }^{1,2,6}$

$$
\underline{\mathbf{W}}\left(\vec{r}_{1}, \vec{r}_{2}, \omega\right)=\left\langle\vec{E}\left(\overrightarrow{r_{1}}, \omega\right) \otimes \vec{E}^{*}\left(\vec{r}_{2}, \omega\right)\right\rangle,
$$

where $\vec{E}\left(\vec{r}_{i}, \omega\right)$ is the electric field of thermal radiation at the position $\vec{r}_{i},\langle\rangle$ indicates the ensemble average, and $*$ denotes complex conjugate. $\underline{\mathbf{W}}$ can be written explicitly in its components as $\mathbf{W}_{\alpha \beta}=\left\langle E_{\alpha} E_{\beta}^{*}\right\rangle$. By its definition, $\underline{\mathbf{W}}$ describes how the radiation fields at two different spatial points correlate. Especially, the spatial coherence of the $\alpha$-component thermal radiation field is described by the variation of $\mathbf{W}_{\alpha \alpha}$ versus the distance between the two field points $\left|\vec{r}_{1}-\vec{r}_{2}\right|$.

On the other hand, the radiation field can relate to the random current sources $\vec{J}$ through the Green's function as $\vec{E}=i \mu_{0} \omega \int_{V} \underline{G} \cdot \vec{J}$, where $\mu_{0}$ is the permeability in vacuum. Moreover, the random current should fulfill fluctuationdissipation theorem: ${ }^{35}$

$$
\begin{aligned}
& \left\langle\vec{J}\left(\overrightarrow{r_{1}}, \omega\right) \otimes \vec{J}^{*}\left(\vec{r}_{2}, \omega\right)\right\rangle \\
& \quad=4 \pi \omega \varepsilon_{0} \varepsilon^{\prime \prime}(\omega) O(\omega, T) O(\omega, T) \delta^{3}\left(\vec{r}_{1}-\overrightarrow{r_{2}}\right) \underline{I},
\end{aligned}
$$

where $\underline{I}$ is the unit tensor, $\varepsilon_{0}$ is the permittivity (permeability) in vacuum, $\varepsilon^{\prime \prime}(\omega)$ is the imaginary part of relative permittivity, $O(\omega, T)$ gives the mean energy of a quantum oscillator at temperature $T$. By using the above two facts and performing Fourier transformation, the cross-spectral density tensor can be written in a very general form in terms of the Green's function in momentum space as

$$
\begin{aligned}
\underline{\mathbf{W}}\left(\vec{r}_{1}, \vec{r}_{2}, \omega\right)= & \underline{\mathbf{W}}\left(\vec{R}_{1}, \vec{R}_{2}, z, \omega\right) \\
= & 4 \pi \omega^{3} \mu_{0}^{2} \varepsilon_{0} \varepsilon^{\prime \prime}(\omega) O(\omega, T) \int_{0}^{h} d z^{\prime} \int_{-\infty}^{\infty} \frac{d^{2} \kappa}{4 \pi^{2}} \\
& \times e^{i \vec{\kappa} \cdot\left(\vec{R}_{1}-\vec{R}_{2}\right)} \underline{\mathbf{g}}\left(\vec{\kappa}, z, z^{\prime}, \omega\right) \underline{\mathbf{g}}^{\dagger}\left(\vec{\kappa}, z, z^{\prime}, \omega\right) .
\end{aligned}
$$

The Green's function $g$ contains the information of the scattering of the radiation field generated by the random current sources. For a TI slab, the introduction of Hall surface states is crucial and brings the mode conversions at the TI surfaces. To take the complex scattering processes into account, we follow the mode expansion scheme to obtain a very general form of the Green's function for the slab geometry.

For the slab geometry (see Fig. 2), the electromagnetic field in each region can be expanded into the eigenfunctions of the Maxwell's equations. The eigenmodes in the bulk of TI slab (region II) should be identical to those in a dielectric slab. Here we have to account the effects of random current sources in the systems. By using the following form: ${ }^{28}$

$$
\vec{J}(\vec{r})=\int_{0}^{h} d z_{0} \int \frac{d \vec{\kappa}}{4 \pi^{2}} \delta\left(z-z_{0}\right) \vec{J}_{\vec{\kappa}} \exp (i \vec{\kappa} \cdot \vec{R}),
$$

the random sources can be converted to plane sources, which would benefit the later mode expansions. Here we have assumed the thickness of the slab is $h$. Therefore, in terms of the transversal electric (TE) modes and transversal magnetic (TM) modes the electric and the magnetic fields in region II
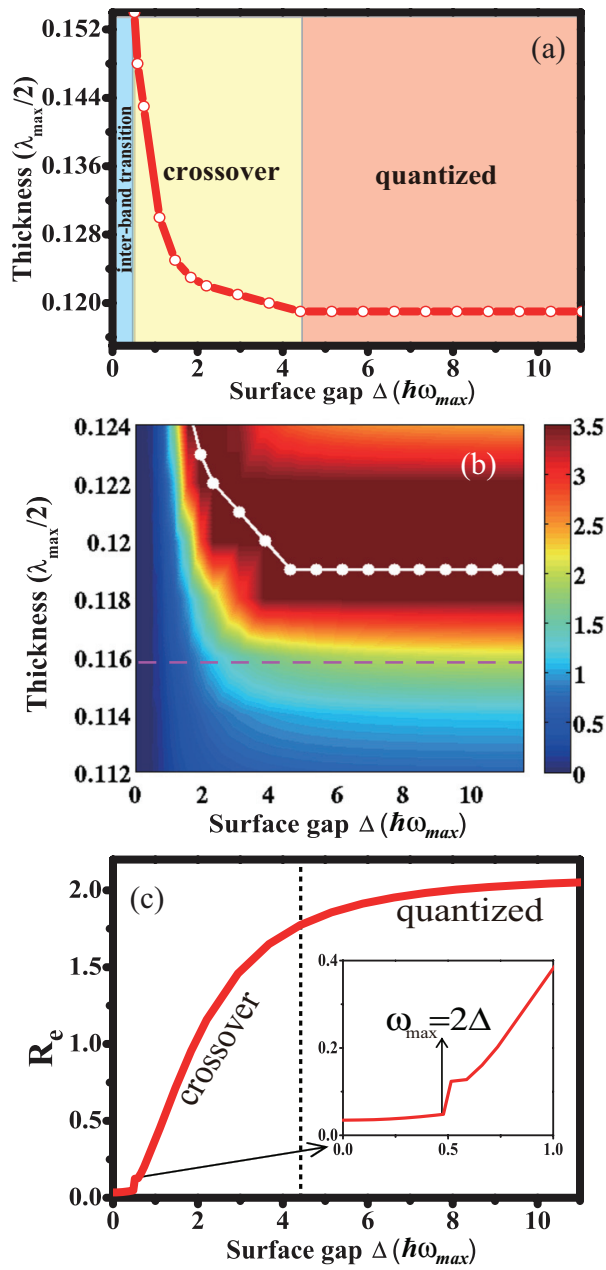

FIG. 2. (Color online) (a) Positions of the resonance induced by the coupling of the surface states and the waveguide modes on the $h-$ $\Delta$ plane: only when $h$ and $\Delta$ fulfill the relation presented by the curve can the resonance can be induced. (b) Plot of the ratio $\mathbf{R}_{e}$ as a function of $h$ and $\Delta: \mathbf{R}_{e}$ depicts the ratio of the TM and TE modes in the radiation field. Here the white curve is to denote the positions of the resonances within the thickness regime. (c) $\mathbf{R}_{e}$ versus $\Delta$ for a TI slab of a thickness $h=0.116 \lambda_{\max } / 2$ [highlighted by pink dash line in (b)].

should be written as

$$
\begin{aligned}
& \vec{E}_{\mathrm{II}}(\varpi, \vec{\kappa}) \\
& =\left[\vec{E}_{s, g,+} \exp \left(-i k_{z, \mathrm{TI}} z_{0}\right)+\vec{E}_{p, g,+} \exp \left(-i k_{z, \mathrm{TI}} z_{0}\right)\right] \\
& \times \Theta\left(z_{0}-z\right) \exp (i \vec{\kappa} \cdot \vec{R}) \\
& +\left[\vec{E}_{s, b,+}+\vec{E}_{p, b,+}+\vec{E}_{s, b,-}+\vec{E}_{p, b,-}\right] \exp (i \vec{\kappa} \cdot \vec{R}) \\
& +\left[\vec{E}_{s, g,-} \exp \left(i k_{z, \mathrm{TI}} z_{0}\right)+\vec{E}_{p, g,-} \exp \left(i k_{z, \mathrm{TI}} z_{0}\right)\right] \\
& \times \Theta\left(z_{0}-z\right) \exp (i \vec{\kappa} \cdot \vec{R})+\overrightarrow{\mathbb{E}} \delta\left(z-z_{0}\right) \exp (i \vec{\kappa} \cdot \vec{R}), \\
& \vec{H}_{\mathrm{II}}(\varpi, \vec{\kappa}) \\
& =\left[\vec{H}_{s, g,+} \exp \left(-i k_{z, \mathrm{TI}} z_{0}\right)+\vec{H}_{p, g,+} \exp \left(-i k_{z, \mathrm{TI}} z_{0}\right)\right] \\
& \times \Theta\left(z_{0}-z\right) \exp (i \vec{\kappa} \cdot \vec{R}) \\
& +\left[\vec{H}_{s, b,+}+\vec{H}_{p, b,+}+\vec{H}_{s, b,-}+\vec{H}_{p, b,-}\right] \exp (i \vec{\kappa} \cdot \vec{R}) \\
& +\left[\vec{H}_{s, g,-} \exp \left(i k_{z, \mathrm{TI}} z_{0}\right)+\vec{H}_{p, g,-} \exp \left(i k_{z, \mathrm{TI}} z_{0}\right)\right] \\
& \times \Theta\left(z_{0}-z\right) \exp (i \vec{\kappa} \cdot \vec{R})+\mu \overrightarrow{\mathbb{B}} \delta\left(z-z_{0}\right) \exp (i \vec{\kappa} \cdot \vec{R}),
\end{aligned}
$$


where $\varpi=\omega / c$ is the reduced angular frequency, $\mu$ is the relative permeability of the slab, $\vec{E}_{s, g, \pm}$ is the electric field of the upward (downward) propagating TE radiation modes generated from the source plane (denoted by the red line shown in Fig. 2), $\vec{E}_{p, g, \pm}$ is the electric field of the upward (downward) propagating TM radiation modes generated from the source plane, $\vec{E}_{s(p), b,+}$ is the electric field of the TE (TM) modes propagating from the lower surface of the slab to the upper one, and $\vec{E}_{s(p), g,-}$ is the electric field of the TE (TM) radiation modes propagating from the upper surface of the slab to the lower one, and $\vec{H}_{s, g, \pm}, \vec{H}_{p, g, \pm}, \vec{H}_{s, b, \pm}$, and $\vec{H}_{p, g, \pm}$ are the corresponding magnetic fields. The explicit form of these quantities are presented below:

$$
\begin{aligned}
\vec{E}_{s, g(b), \pm} & =E_{s, g(b), \pm} \exp \left( \pm i k_{z, \mathrm{TI}} z\right) \hat{s} \\
\vec{E}_{p, g(b), \pm} & =E_{p, g(b), \pm} \exp \left( \pm i k_{z, \mathrm{TI}} z\right) \hat{p}_{ \pm}, \\
\vec{H}_{s, g(b), \pm} & =\sqrt{\frac{\varepsilon}{\mu}} E_{s, g(b), \pm} \exp \left( \pm i k_{z, \mathrm{TI}} z\right) \hat{s}, \\
\vec{H}_{p, g(b), \pm} & =-\sqrt{\frac{\varepsilon}{\mu}} E_{p, g(b), \pm} \exp \left( \pm i k_{z, \mathrm{TI}} z\right) \hat{p}_{ \pm} .
\end{aligned}
$$

Here $\hat{s}$ is the unit vector of TE modes for the given $\vec{\kappa}$ and $\varpi$, and $\hat{p}_{ \pm}$are the unit vectors of TM modes propagating upward (downward). Following the similar scheme, the radiation field in regions I and III can be written as

$$
\begin{aligned}
& \vec{E}_{\mathrm{I}}(\varpi, \vec{\kappa})=\left(E_{s,-} \hat{s}+E_{p,-} \hat{p}_{-}\right) \exp (i \vec{\kappa} \cdot \vec{R}) \exp \left(-i k_{z, \mathrm{I}} z\right), \\
& \vec{H}_{\mathrm{I}}(\varpi, \vec{\kappa})=\left(E_{p,-} \hat{s}-E_{s,-} \hat{p}_{-}\right) \exp (i \vec{\kappa} \cdot \vec{R}) \exp \left(-i k_{z, \mathrm{I}} z\right), \\
& \vec{E}_{\mathrm{III}}(\varpi, \vec{\kappa})=\left(E_{s,+} \hat{s}+E_{p,+} \hat{p}_{+}\right) \exp (i \vec{\kappa} \cdot \vec{R}) \exp \left(i k_{z, \mathrm{III}} z\right), \\
& \vec{H}_{\mathrm{III}}(\varpi, \vec{\kappa})=\left(E_{p,+} \hat{s}-E_{s,+} \hat{p}_{+}\right) \exp (i \vec{\kappa} \cdot \vec{R}) \exp \left(i k_{z, \mathrm{III}} z\right)
\end{aligned}
$$

where $E_{p,-}$ is the amplitude of TM modes in region I, $E_{s,-}$ is the amplitude of TE modes in region I, $E_{p,+}$ is the amplitude of TM modes in region III, and $E_{s,+}$ is the amplitude of TE modes in region III.

As the radiation field scatters on the surfaces of the TI slab, the current bounding on the surface would be generated. For a TE (TM) mode with the given $\varpi$ and $\kappa$, the corresponding current is given by

$$
\begin{aligned}
& j_{s}^{\mathrm{IIII}} \hat{s}=\sigma_{x x}\left(\vec{E}_{s, b,+}^{T}+\vec{E}_{s, b,-}^{T}+\vec{E}_{s, g,-}^{T} \exp \left(i k_{z, \mathrm{TI}} z_{0}\right)\right)+\sigma_{x y} \hat{z} \times\left(\vec{E}_{p, b,+}^{T}+\vec{E}_{p, b,-}^{T}+\vec{E}_{p, g,-}^{T} \exp \left(i k_{z, \mathrm{TI}} z_{0}\right)\right), \\
& j_{p}^{\mathrm{I}, \mathrm{II}} \hat{p}_{+}=\sigma_{x x}\left(\vec{E}_{p, b,+}^{T}+\vec{E}_{p, b,-}^{T}+\vec{E}_{p, g,-}^{T} \exp \left(i k_{z, \mathrm{TI}} z_{0}\right)\right)+\sigma_{x y} \hat{z} \times\left(\vec{E}_{s, b,+}^{T}+\vec{E}_{s, b,-}^{T}+\vec{E}_{s, g,-}^{T} \exp \left(i k_{z, \mathrm{TI}} z_{0}\right)\right), \\
& j_{s}^{\mathrm{II}, \mathrm{III}} \hat{s}=\sigma_{x x}\left(\vec{E}_{s, b,+}^{T}+\vec{E}_{s, b,-}^{T}+\vec{E}_{s, g,+}^{T} \exp \left(-i k_{z, \mathrm{TI}} z_{0}\right)\right)+\sigma_{x y} \hat{z} \times\left(\vec{E}_{p, b,+}^{T}+\vec{E}_{p, b,-}^{T}+\vec{E}_{p, g,+}^{T} \exp \left(-i k_{z, \mathrm{TI}} z_{0}\right)\right), \\
& j_{p}^{\mathrm{II}, \mathrm{III}} \hat{p}_{-}=\sigma_{x x}\left(\vec{E}_{p, b,+}^{T}+\vec{E}_{p, b,-}^{T}+\vec{E}_{p, g,+}^{T} \exp \left(-i k_{z, \mathrm{TI}} z_{0}\right)\right)+\sigma_{x y} \hat{z} \times\left(\vec{E}_{s, b,+}^{T}+\vec{E}_{s, b,-}^{T}+\vec{E}_{s, g,+}^{T} \exp \left(-i k_{z, \mathrm{TI}} z_{0}\right)\right),
\end{aligned}
$$

where $J_{s,(p)}^{\mathrm{I}, \mathrm{II}}$ is the magnitude of the current in the $\hat{s}\left(\hat{p}_{ \pm}\right)$direction on the interface between regions I and II, $J_{s,(p)}^{\mathrm{II}, \mathrm{III}}$ is the magnitude of the current in the $\hat{s}\left(\hat{p}_{ \pm}\right)$direction on the interface between regions II and III, and the subscript $T$ denotes the components parallel to the interfaces.

By using the boundary conditions containing surface currents, we would have equations determining the radiation field in regions I and III. These equations are very long, and here we only present the radiation fields in region III solved from these equations:

$$
\begin{aligned}
E_{p,+} & =T_{p,+; s, g,+} E_{s, g,+}+T_{p,+; s, g,-} E_{s, g,-}+T_{p,+; p, g,+} E_{p, g,+}+T_{p,+; p, g,-} E_{s, g,-}, \\
E_{s,+} & =T_{s,+; s, g,+} E_{s, g,+}+T_{s,+; s, g,-} E_{s, g,-}+T_{s,+; p, g,+} E_{p, g,+}+T_{s,+; p, g,-} E_{s, g,-},
\end{aligned}
$$

where $T_{p,+; s(p), g, \pm}$ is the magnitude of the TM modes in region III converted from the upward (downward) propagating TE (TM) modes generated from the random sources, $T_{s,+; s(p), g, \pm}$ is the magnitude of the TE modes in region III converted from the upward (downward) propagating TE (TM) modes generated from the random sources (for the detailed expressions of these coefficients, please see Appendix B). By using the definition of the Green function $\vec{E}_{\mathrm{III}}(\varpi, \vec{\kappa})=\frac{g(\varpi, \vec{\kappa}) \cdot \vec{J}_{\varpi, \vec{\kappa}}}{i \omega \mu_{0}}$, we can obtain the general form of the Green's function for the slab geometry as

$$
\begin{aligned}
\underline{\mathbf{g}}\left(\vec{\kappa}, z_{0}, z_{1}, \omega\right)= & {\left[\frac{i T_{p,+; s, g,+}}{2 k_{z, \mathrm{TI}}} \hat{p}_{+}^{V} \hat{s}^{\mathrm{TI}}+\frac{i T_{p,+; s, g,-}}{2 k_{z, \mathrm{TI}}} \hat{p}_{+}^{V} \hat{s}^{\mathrm{TI}}+\frac{i T_{p,+; p, g,+}}{2 k_{z, \mathrm{TI}}} \hat{p}_{+}^{V} \hat{p}_{+}^{\mathrm{TI}}+\frac{i T_{p,+; p, g,-}}{2 k_{z, \mathrm{TI}}} \hat{p}_{+}^{V} \hat{p}_{-}^{\mathrm{TI}}\right] e^{i k_{z, v} z z_{1}} } \\
& +\left[\frac{i T_{s,+; s, g,+}}{2 k_{z, \mathrm{TI}}} \hat{s}^{V} \hat{s}^{\mathrm{TI}}+\frac{i T_{s,+; s, g,-}}{2 k_{z, \mathrm{TI}}} \hat{s}^{V} \hat{s}^{\mathrm{TI}}+\frac{i T_{s,+; p, g,+}}{2 k_{z, \mathrm{TI}}} \hat{s}^{V} \hat{p}_{+}^{\mathrm{TI}}+\frac{i T_{s,+; p, g,-}}{2 k_{z, \mathrm{TI}}} \hat{s}^{V} \hat{p}_{-}^{\mathrm{TI}}\right] e^{i k_{z, v} z_{1}} .
\end{aligned}
$$

The Green's function relates the radiation field on the plane $z=z_{1}$ in region III to the source plane located at $z=z_{0}$ in the TI slab for a given angular frequency $\omega$ and a given tangential wave vector $\vec{\kappa}$ (a wave vector in the $x-y$ plane). 
By inserting the Green's function into the expression of the cross-spectral density tensor (7), one can obtain the concrete form of the cross-spectral density tensor. The concrete expressions of nonvanishing elements are presented below:

$$
\begin{aligned}
\mathbf{W}_{x x} & \left(\vec{R}_{1}, \vec{R}_{2}, z, \omega\right) \\
= & 4 \pi \omega^{3} \mu_{0}^{2} \varepsilon_{0} \varepsilon^{\prime \prime}(\omega) O(\omega, T) \int_{0}^{h} d z^{\prime} \int_{0}^{\infty} \frac{\kappa d \kappa}{2 \pi} \frac{e^{i\left(k_{z, V}-k_{z, v}^{*}\right)}}{\left|2 k_{z, \mathrm{TI}}\right|^{2}} \frac{\left|k_{z, V}\right|^{2}}{\varpi^{2}}\left(J_{0}(\kappa x)-\frac{J_{1}(\kappa x)}{\kappa x}\right) \\
& \times\left(\left|T_{p,+; s, g,+}\right|^{2}+\left|T_{p,+; s, g,-}\right|^{2}+2 \operatorname{Re}\left(T_{p,+; s, g,+} T_{p,+; s, g,-}\right)+C_{1}\left(\left|T_{p,+; p, g,+}\right|^{2}+\left|T_{p,+; p, g,-}\right|^{2}\right)+2 C_{2} \operatorname{Re}\left(T_{p,+; p, g,+} T_{p,+; p, g,-}\right)\right) \\
& +4 \pi \omega^{3} \mu_{0}^{2} \varepsilon_{0} \varepsilon^{\prime \prime}(\omega) O(\omega, T) \int_{0}^{h} d z^{\prime} \int_{0}^{\infty} \frac{\kappa d \kappa}{2 \pi} \frac{J_{1}(\kappa x)}{\kappa x} \frac{e^{i\left(k_{z, V}-k_{z, v}^{*}\right)}}{\left|2 k_{z, \mathrm{TI}}\right|^{2}} \\
& \times\left(\left|T_{s,+; s, g,+}\right|^{2}+\left|T_{s,+; s, g,-}\right|^{2}+2 \operatorname{Re}\left(T_{s,+; s, g,+} T_{s,+; s, g,-}\right)+C_{1}\left(\left|T_{s,+; p, g,+}\right|^{2}+\left|T_{s,+; p, g,-}\right|^{2}\right)+2 C_{2} \operatorname{Re}\left(T_{s,+; p, g,+} T_{s,+; p, g,-}\right)\right),
\end{aligned}
$$

$$
\begin{aligned}
\mathbf{W}_{y y} & \left(\vec{R}_{1}, \vec{R}_{2}, z, \omega\right) \\
= & 4 \pi \omega^{3} \mu_{0}^{2} \varepsilon_{0} \varepsilon^{\prime \prime}(\omega) O(\omega, T) \int_{0}^{h} d z^{\prime} \int_{0}^{\infty} \frac{\kappa d \kappa}{2 \pi} \frac{e^{i\left(k_{z, V}-k_{z, V}^{*}\right) z}}{\left|2 k_{z, \mathrm{TI}}\right|^{2}} \frac{\left|k_{z, V}\right|^{2}}{\varpi^{2}} \frac{J_{1}(\kappa x)}{\kappa x} \\
& \times\left(\left|T_{p,+; s, g,+}\right|^{2}+\left|T_{p,+; s, g,-}\right|^{2}+2 \operatorname{Re}\left(T_{p,+; s, g,+} T_{p,+; s, g,-}\right)+C_{1}\left(\left|T_{p,+; p, g,+}\right|^{2}+\left|T_{p,+; p, g,-}\right|^{2}\right)+2 C_{2} \operatorname{Re}\left(T_{p,+; p, g,+} T_{p,+; p, g,-}\right)\right) \\
& +4 \pi \omega^{3} \mu_{0}^{2} \varepsilon_{0} \varepsilon^{\prime \prime}(\omega) O(\omega, T) \int_{0}^{h} d z^{\prime} \int_{0}^{\infty} \frac{\kappa d \kappa}{2 \pi}\left(J_{0}(\kappa x)-\frac{J_{1}(\kappa x)}{\kappa x}\right) \frac{e^{i\left(k_{z, V}-k_{z, V}^{*}\right) z}}{\left|2 k_{z, \mathrm{TI}}\right|^{2}} \\
& \times\left(\left|T_{s,+; s, g,+}\right|^{2}+\left|T_{s,+; s, g,-}\right|^{2}+2 \operatorname{Re}\left(T_{s,+; s, g,+} T_{s,+; s, g,-}\right)+C_{1}\left(\left|T_{s,+; p, g,+}\right|^{2}+\left|T_{s,+; p, g,-}\right|^{2}\right)+2 C_{2} \operatorname{Re}\left(T_{s,+; p, g,+} T_{s,+; p, g,-}\right)\right)
\end{aligned}
$$

$$
\begin{aligned}
\mathbf{W}_{z z} & \left(\vec{R}_{1}, \vec{R}_{2}, z, \omega\right) \\
= & 4 \pi \omega^{3} \mu_{0}^{2} \varepsilon_{0} \varepsilon^{\prime \prime}(\omega) O(\omega, T) \int_{0}^{h} d z^{\prime} \int_{0}^{\infty} \frac{\kappa d \kappa}{2 \pi} \frac{e^{i\left(k_{z, V}-k_{z, V}^{*}\right) z}}{\left|2 k_{z, \mathrm{TI}}\right|^{2}} \frac{\left|k_{z, V}\right|^{2}}{\omega^{2}} J_{0}(\kappa x) \\
& \times\left(\left|T_{p,+; s, g,+}\right|^{2}+\left|T_{p,+; s, g,-}\right|^{2}+2 \operatorname{Re}\left(T_{p,+; s, g,+} T_{p,+; s, g,-}\right)+C_{1}\left(\left|T_{p,+; p, g,+}\right|^{2}+\left|T_{p,+; p, g,-}\right|^{2}\right)+2 C_{2} \operatorname{Re}\left(T_{p,+; p, g,+} T_{p,+; p, g,-}\right)\right),
\end{aligned}
$$

$$
\begin{aligned}
\mathbf{W}_{x y}\left(\vec{R}_{1}, \vec{R}_{2}, z, \omega\right)= & 4 \pi \omega^{3} \mu_{0}^{2} \varepsilon_{0} \varepsilon^{\prime \prime}(\omega) O(\omega, T) \int_{0}^{h} d z^{\prime} \int_{0}^{\infty} \frac{\kappa d \kappa}{2 \pi} \frac{e^{i\left(k_{z, V}-k_{z, v}^{*}\right) z}}{\left|2 k_{z, \mathrm{TI}}\right|^{2}} \frac{k_{z, V}^{*}}{\varpi}\left(J_{0}(\kappa x)-\frac{J_{1}(\kappa x)}{\kappa x}\right) \\
& \times\left(T_{s,+; s, g,+} T_{p,+; s, g,+}^{*}+T_{s,+; s, g,+} T_{p,+; s, g,-}^{*}+T_{s,+; s, g,-} T_{p,+; s, g,+}^{*}+T_{s,+; s, g,-} T_{p,+; s, g,-}^{*}\right. \\
& \left.+C_{1}\left(T_{s,+; p, g,+} T_{p,+; p, g,+}^{*}+T_{s,+; p, g,-} T_{p,+; p, g,-}^{*}\right)+C_{2}\left(T_{s,+; p, g,+} T_{p,+; p, g,-}^{*}+T_{s,+; p, g,-} T_{p,+; p, g,+}^{*}\right)\right) \\
& +4 \pi \omega^{3} \mu_{0}^{2} \varepsilon_{0} \varepsilon^{\prime \prime}(\omega) O(\omega, T) \int_{0}^{h} d z^{\prime} \int_{0}^{\infty} \frac{\kappa d \kappa}{2 \pi} \frac{e^{i\left(k_{z, V}-k_{z, V}^{*}\right) z}}{\left|2 k_{z, \mathrm{TI}}\right|^{2}} \frac{k_{z, V}}{\varpi} \frac{J_{1}(\kappa x)}{\kappa x} \\
& \times\left(T_{s,+; s, g,+}^{*} T_{p,+; s, g,+}+T_{s,+; s, g,+}^{*} T_{p,+; s, g,-}+T_{s,+; s, g,-}^{*} T_{p,+; s, g,+}+T_{s,+; s, g,-}^{*} T_{p,+; s, g,-}\right. \\
& \left.+C_{1}\left(T_{s,+; p, g,+}^{*} T_{p,+; p, g,+}+T_{s,+; p, g,-}^{*} T_{p,+; p, g,-}\right)+C_{2}\left(T_{s,+; p, g,+}^{*} T_{p,+; p, g,-}+T_{s,+;, g,-}^{*} T_{p,+;, g,+}\right)\right),
\end{aligned}
$$

where $C_{1}=\frac{\left|k_{z, \pi \mathrm{TI}}\right|^{2}+\kappa^{2}}{\omega^{2} \varepsilon \mu} C_{2}=\frac{-\left|k_{z, \mathrm{rII}}\right|^{2}+\kappa^{2}}{\omega^{2} \varepsilon \mu}$, and $\mathbf{W}_{x y}=\mathbf{W}_{y x}$ can be viewed as the consequence of the mode conversion between the TE and TM modes at the TI surfaces. From these expressions, we notice that $\mathbf{W}_{z z}$ contains only the TM contribution, which is due to the fact that only the TM modes have the $z$ component.

\section{THERMAL COHERENCE PROPERTIES OF A TI SLAB}

In the section, we will analyze the nonvanishing elements of the cross-spectral density tensor to study the coherence properties of a TI slab. We will demonstrate that the coupling of the gap surface states and the waveguide modes in the TI slab would introduce a novel resonance, which can modify the ratio of the TM and TE components in the radiation field.
This modification can lead to the dramatic change of the coherence properties of the thermal radiation field. To make the discussion concrete, we set the relative permittivity as $\varepsilon=25,{ }^{27}$ with a small dissipation $(0.005 i)$ and the relative permeability $\mu$ as 1 . The temperature of the TI slab is set to be $T=6 \mathrm{~K}\left(k_{B} T \approx 0.0005 \mathrm{eV}\right)$. Without losing the generality, we work at the maximal radiation frequency of the temperature $\left(\hbar \omega_{\max } \approx 0.0026 \mathrm{eV}\right)$. These parameters are assumed throughout the study of the section.

\section{A. A novel resonance and mode conversion at the TI surfaces}

For a TI slab, the eigenmodes in the bulk should be the waveguide modes, which has no difference from a normal dielectric slab, while except for those the surface states on the TI surfaces can couple to the waveguide modes in the 
bulk through the scattering of the radiation fields on the TI surfaces. As the coupling between them is strong, a novel resonance depending on both the thickness of the slab $h$ and the surface gap $\Delta$ would be induced. To demonstrate the origin of the resonance explicitly, we consider the denominators of the transmission coefficients, which are defined in last section (the concrete forms are presented in Appendix A). The denominators of $T_{p,+; p, g,+}, T_{p,+; p, g,-}, T_{p,+; s, g,+}$, and $T_{p,+; s, g,-}$, which correspond to the transmission channels leading to the TM modes in vacuum, are the same and given by

$$
D_{p}=\left(1+\Theta_{p}\right)^{2}-\left(r_{p}-r_{s} \Theta_{p}\right)^{2} e^{i 2 k_{z, \mathrm{TI}} h},
$$

where

$$
\begin{aligned}
r_{s} & =\frac{\sqrt{1 / \varepsilon}-\beta+\frac{4 \pi \sigma_{x x} k_{z, V}}{\omega} \sqrt{\varepsilon}}{\sqrt{1 / \varepsilon}+\beta+\frac{4 \pi \sigma_{x x} k_{z, V}}{\omega} \sqrt{\varepsilon}}, \\
r_{p} & =\frac{1-\sqrt{\varepsilon} \beta-\frac{4 \pi \sigma_{x x} \omega}{k_{z, V} c^{2}}}{1+\sqrt{\varepsilon} \beta+\frac{4 \pi \sigma_{x x} \omega}{k_{z, V} c^{2}}},
\end{aligned}
$$

and $\beta=k_{z, V} \sqrt{\varepsilon} / k_{z, \mathrm{TI}}$. Similarly, The denominators of $T_{s,+; p, g,+}, T_{s,+; p, g,-}, T_{s,+; s, g,+}$, and $T_{s,+; s, g,-}$, which correspond to the transmission channels leading to the TE modes in vacuum, are also the same and given by

$$
D_{s}=\left(1+\Theta_{s}\right)^{2}-\left(r_{s}-r_{p} \Theta_{s}\right)^{2} e^{i 2 k_{z, \mathrm{TI}} h} .
$$

In the above, the expressions of $\Theta_{p}$ and $\Theta_{s}$ are too long, and we present them in Appendix A.

As the surface states are removed, $\sigma_{x x}, \Theta_{s}$, and $\Theta_{p}$ are 0 , and thus the above expressions reduce to the denominators of the transmission coefficients for the TE and TM modes through a dielectric slab. As it is well known, the zeros of the denominators present the dispersion of the waveguide modes in such a dielectric slab. For a TI slab, the additional terms due to the nonzero $\sigma_{x x}, \Theta_{s}$, and $\Theta_{p}$ can be viewed as the consequence of the coupling of the surface states and the waveguide modes through the scattering of the radiation fields on the TI surfaces.

To see the effects of such a coupling clearly, we assume that the thickness of the TI slab is smaller than $\frac{\pi}{\sigma \sqrt{\varepsilon_{T I}-\varepsilon_{V}}} \approx$ $0.2(\lambda / 2)$ so that only the fundamental waveguide modes exist in the bulk. When the surface gap $\Delta$ is smaller than the photon energy of the radiation field, we find that both $D_{p}$ and $D_{s}$ have no zero points within the regime of slab thickness, which indicates that the coupling between the surface states and the waveguide modes is very weak. This parameter regime on the $h-\Delta$ plane is the interband transition regime, which is highlighted in blue color in Fig. 2(a). As the surface gap is larger than the photon energy of the radiation field, zeros can be found in $D_{p}$ for a proper thickness, which means that the coupling between the surface states and the waveguide modes can induce a novel resonance to the transmission channels leading to the TM modes in vacuum. As $2 \Delta$ is larger but comparable with $\hbar \omega(2 \Delta \gtrsim \hbar \omega)$, the thickness $h$, for which the resonance can be induced, decreases with the increasing of the surface gap $\Delta$. This parameter regime is the crossover regime, which is highlighted in yellow color in Fig. 2(a). When $\Delta$ is much larger than $\hbar \omega(\Delta \gg \hbar \omega)$, the thickness $h$, for which the resonance can be induced, is fixed at $0.119 \lambda_{\max } / 2$ and independent of the increasing of the surface gap. This parameter regime is the half integer quantized surface Hall conductivity (HIQSHC) regime, which is highlighted in red in Fig. 2(a).

Since the novel resonance is introduced to all transmission channels leading to the TM modes in vacuum, the ratio of the TM and TE modes in the radiation field in vacuum would change accordingly. To present the point clearly, we introduce a quantity to characterize the ratio:

$$
\mathbf{R}_{e}=\frac{\mathbf{W}_{x x, p}\left(\vec{R}_{1}-\vec{R}_{2}=\overrightarrow{0}\right)+\mathbf{W}_{y y, p}\left(\vec{R}_{1}-\vec{R}_{2}=\overrightarrow{0}\right)}{\mathbf{W}_{x x, s}\left(\vec{R}_{1}-\vec{R}_{2}=\overrightarrow{0}\right)+\mathbf{W}_{y y, s}\left(\vec{R}_{1}-\vec{R}_{2}=\overrightarrow{0}\right)},
$$

where $\mathbf{W}_{x x, p(s)}(\overrightarrow{0})$ is the energy of the $x$-component radiation field contributed by the TM (TE) modes, and $\mathbf{W}_{y y, p(s)}(\overrightarrow{0})$ is the energy of the $y$-component radiation field contributed by the TM (TE) modes. Thus, $\mathbf{R}_{e}$ describes the ratio of the TM and TE modes in the tangential components of the radiation field. We plot the ratio $\mathbf{R}_{e}$ as a function of a function of slab thickness $(h)$ and surface gap $(\Delta)$ in Fig. 2(b). The calculation is conducted for the radiation field on the plane of a height $z=\lambda_{\max } / 20$ $\left(\lambda_{\max } \approx 477 \mu \mathrm{m}\right)$ above the upper TI/vacuum interface. It can be seen that $\mathbf{R}_{e}$ is much larger in the vicinity of the resonant states, which is denoted by the white curve with symbols.

As an example of a TI slab of a specified thickness, we set the slab thickness as $h=0.116 \lambda_{\max } / 2$ so that it is in the vicinity of the resonant thickness $\left(h=0.119 \lambda_{\max } / 2\right)$ in the HIQSHC regime. Then we plot the ratio $\mathbf{R}_{e}$ as the function of the surface gap $\Delta$ in Fig. 2(c). We find that the variations of $\mathbf{R}_{e}$ versus $\Delta$ in the three regimes mentioned above are quite different: (1) as $2 \Delta \lesssim \hbar \omega$, the system is in the interband transition regime, and the ratio $\mathbf{R}_{e}$ is very small, which means the TE modes dominate the tangential components in the radiation field; (2) as $2 \Delta \gtrsim \hbar \omega$, the system enters the crossover regime, and the ratio increases sensitively with the increasing of the surface gap $\Delta$; (3) as $\Delta \gg \hbar \omega$, the system is in the HIQSHC regime, and the ratio approaches the maximal values and manifests a plateau.

\section{B. Spatial coherence of the thermal radiation field}

The change of the ratio of the TM and TE modes in the radiation field would have great influence on the spatial coherence of the thermal radiation field. As shown in Appendix B, the TM and TE radiation modes exhibit quite different spatial coherence behaviors. Therefore, with the variation of $\mathbf{R}_{e}$, one can expect that the spatial coherence of the thermal radiation field should change accordingly. To demonstrate the point explicitly, we consider a TI slab of a thickness $h=0.116 \lambda_{\max } / 2$ and calculate the nonvanishing elements of the cross-spectral density tensor by probing the radiation field on the plane $\lambda_{\max } / 20$ above the upper TI/vacuum interface along the $x$ axis (see Fig. 1).

In Fig. 3(a), we first show the spatial coherence of $\mathbf{W}_{x x}$ at four different surface gaps. For a comparison, we also show the spatial coherence of $\mathbf{W}_{x x}$ for the dielectric slab with the same permittivity, permeability, and thickness [black curve in Fig. 3(a)]. In Fig. 3(a), with the increasing surface gap, the spatial coherence of $\mathbf{W}_{x x}$ increases accordingly until the system enters the HIQSHC regime. We also notice that the spatial coherence of $\mathbf{W}_{x x}$ at $\Delta=0 \mathrm{eV}$ is almost the same 

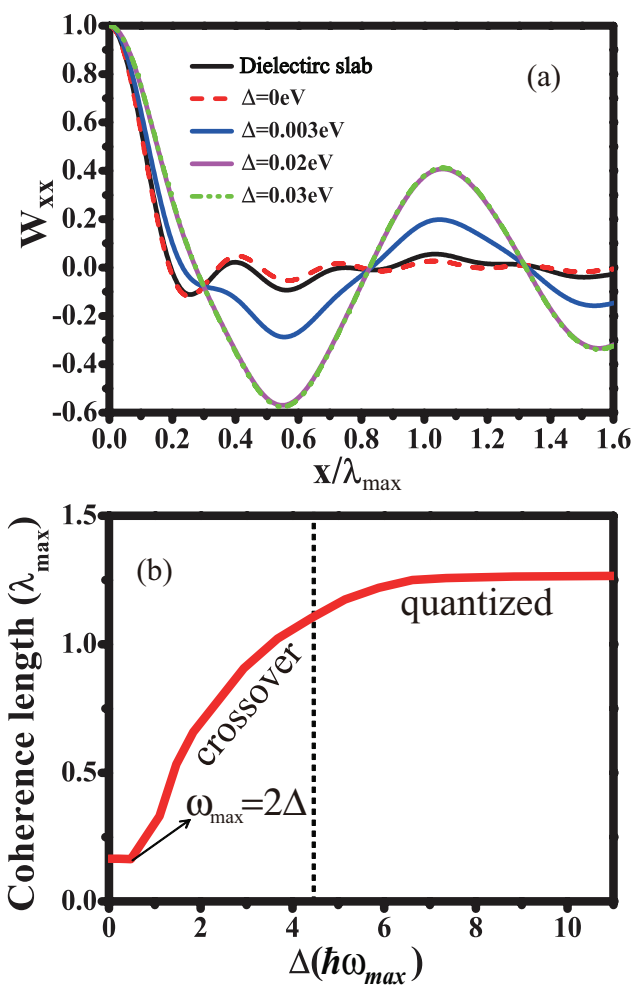

FIG. 3. (Color online) (a) $\mathbf{W}_{x x}\left(\left|\vec{R}_{1}-\vec{R}_{2}\right|\right)$ versus $x=\left|\vec{R}_{1}-\vec{R}_{2}\right|$ for various surface gaps; the black curve is $\mathbf{W}_{x x}\left(\left|\vec{R}_{1}-\vec{R}_{2}\right|\right)$ for the dielectric slab. (b) Plot of the coherence length of $\mathbf{W}_{x x}\left(\left|\vec{R}_{1}-\vec{R}_{2}\right|\right)$ as a function of the surface gap $\Delta$. In the calculation, we assume that the thickness of the TI slab is $h=0.116 \lambda_{\max } / 2$, and the dielectric slab has the same permittivity, permeability, and thickness as the TI slab.

with that of the dielectric slab with the same permittivity, permeability, and thickness.

To quantitatively characterize the spatial coherence, one may define the coherence length of an element in the crossspectral density tensor $\mathbf{W}_{\alpha \beta}$ as ${ }^{6}$

$$
\ell_{\alpha \beta}(z, \lambda)=\frac{2 \int_{0}^{\infty}\left|\mathbf{W}_{\alpha \beta}(x, z, \lambda)\right|^{2}}{\left|\mathbf{W}_{\alpha \beta}(0, z, \lambda)\right|^{2}},
$$

where $z$ is the distance between the probing plane and the upper TI/vacuum interface, and $\lambda$ is the wavelength under the consideration. For our present case, $z=\lambda_{\max } / 20$, and $\lambda$ corresponds to the wavelength of the maximal radiation frequency of the temperature.

In Fig. 3(b), we plot the coherence length $\ell_{x x}$ of $\mathbf{W}_{x x}$ as a function of the surface gap $\Delta$. In the interband transition regime, the coherence length is very small and does not change very much with the variation of the surface gap. In the crossover regime, the coherence length increases with the increasing of the surface gap very sensitively. As the system enters the HIQSHC regime, the coherence length approaches the maximal value and manifests again a plateau with the increasing of the surface gap. We notice that the coherence length of $\mathbf{W}_{x x}$ for the dielectric slab of the same permittivity, permeability, and thickness is about $0.17 \lambda_{\max }$, which is slightly longer than the coherence length of $\mathbf{W}_{x x}$ for the TI slab in the interband transition regime $\left(\ell_{x x} \sim 0.165 \lambda_{\max }\right)$. In the HIQSHC regime, the coherence length of $\mathbf{W}_{x x}$ is as long
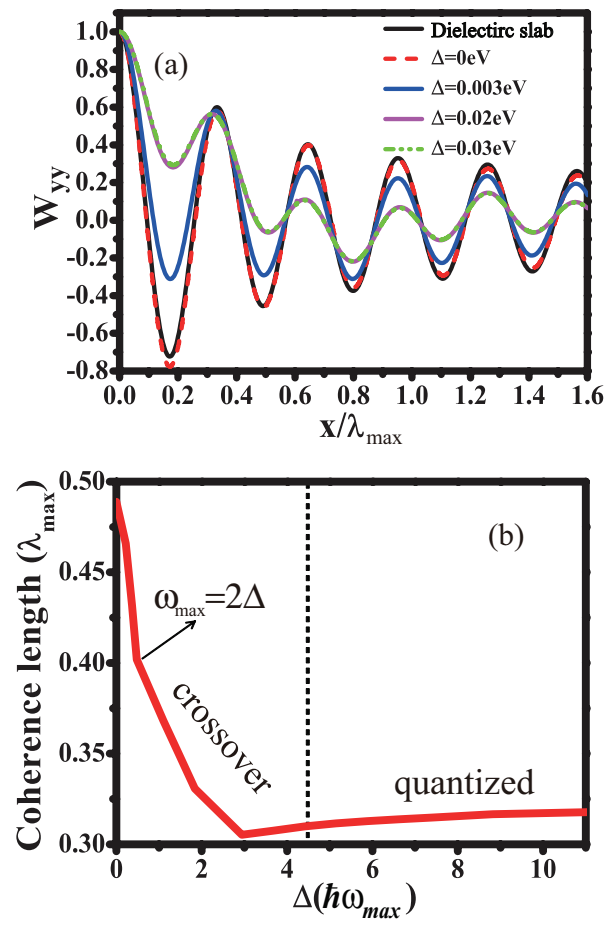

FIG. 4. (Color online) (a) $\mathbf{W}_{y y}\left(\left|\vec{R}_{1}-\vec{R}_{2}\right|\right)$ versus $x=\left|\vec{R}_{1}-\vec{R}_{2}\right|$ for various surface gaps; the black curve is $\mathbf{W}_{y y}\left(\left|\vec{R}_{1}-\vec{R}_{2}\right|\right)$ for the dielectric slab. (b) Plot of the coherence length of $\mathbf{W}_{y y}\left(\left|\vec{R}_{1}-\vec{R}_{2}\right|\right)$ as a function of the surface gap $\Delta$. In the calculation, we assume that the thickness of the TI slab is $h=0.116 \lambda_{\max } / 2$, and the dielectric slab has the same permittivity, permeability, and thickness as the TI slab.

as $\ell_{x x} \sim 1.25 \lambda_{\max }$, which is almost ten times the coherence length of $\mathbf{W}_{x x}$ for the dielectric slab.

Then we show the spatial coherence of $\mathbf{W}_{y y}$ at four different surface gaps in Fig. 4(a). The spatial coherence of $\mathbf{W}_{y y}$ for the dielectric slab of the same permittivity, permeability, and thickness is also shown by the black curve in Fig. 4(a) for a comparison. For a quantitative description of the behavior, we present the coherence length of $\mathbf{W}_{y y}$ as a function of surface gap in Fig. 4(b). From the results shown in Fig. 4, we observe that the coherence length of $\mathbf{W}_{y y}$ deceases with the increasing surface gap. For $\mathbf{W}_{y y}$, the coherence length of the dielectric slab is about $0.49 \lambda_{\max }$, which is also slightly larger than the coherence length of the TI slab in the interband transition regime $\left(\ell_{x x} \sim 0.488 \lambda_{\max }\right)$. The coherence length of $\mathbf{W}_{y y}$ for the TI slab in the HIQSHC regime is about $0.32 \lambda_{\max }$, which is about $2 / 3$ the coherence length of the dielectric slab.

The opposite behaviors of $\mathbf{W}_{x x}$ and $\mathbf{W}_{y y}$ with the increasing surface gap can be understood from the ratio $\mathbf{R}_{e}$. With the increasing of the surface gap, $\mathbf{R}_{e}$ increases, which means the radiation field contains more and more TM components. For $\mathbf{W}_{x x}$, TM modes have a much better coherence than TE modes (see Appendix B). Therefore, the coherence length of $\mathbf{W}_{x x}$ increases with the increasing surface gap. However, for $\mathbf{W}_{y y}$, TE modes have a much better coherence than TM modes (see Appendix B). Consequently, the behavior of $\mathbf{W}_{y y}$ is opposite to that of $\mathbf{W}_{x x}$, when the surface gap increases.

For $\mathbf{W}_{z z}$, it contains only the contribution from TM modes, so the spatial coherence of $\mathbf{W}_{z z}$ would not change with the variation of the surface gap. For this reason, we do not present the 
spatial coherence of $\mathbf{W}_{z z}$ here. On the other hand, the nonzero surface Hall conductivity would induce the nonvanishing off-diagonal element $\mathbf{W}_{x y}\left(\mathbf{W}_{y x}\right)$, which is simply vanishing for a dielectric slab. However, comparing to the diagonal elements, for example $\mathbf{W}_{x x}$, its correlation strength $\left[\left|\mathbf{W}_{x y}(\overrightarrow{0})\right|\right]$ is about $1 / 200$ of that of $\mathbf{W}_{x x}$. Given the weakness of the correlation, we do not discuss the element $\mathbf{W}_{x y}$ here either.

\section{Thermal radiation energy density}

Another important quantity characterizing the thermal radiation field is the thermal radiation energy density, which can be expressed in terms of the cross-spectral density tensor as

$$
\mathbf{U}_{e}\left(z, \omega_{\max }\right)=\frac{\varepsilon_{0}}{2 \pi^{2}} \operatorname{Tr}(\mathbf{W}),
$$

where $\operatorname{Tr}$ denotes the trace of the cross-spectral density tensor, and $\omega_{\max }$ denotes the maximal radiation frequency of the temperature $T=6 \mathrm{~K}$. By using the formula, we calculate the thermal radiation energy density from the TI slab of a thickness $h=0.116 \lambda_{\max } / 2$. For the easy comparison with the results from other systems, we have normalized the results to the black-body radiation energy density for a given frequency $\frac{2 \omega^{2}}{\pi c} O\left(\omega_{\max }, T\right)$.

In Fig. 5(a), we plot $\mathbf{U}_{e}$ as the function of $\Delta$ by probing the radiation field on the plane $z=\lambda_{\max } / 20$ above the upper $\mathrm{TI} /$ vacuum interface along the $x$ axis. We notice that the variation of $\mathbf{U}_{e}$ versus $\Delta$ is very similar to that of $\mathbf{R}_{e}$. In
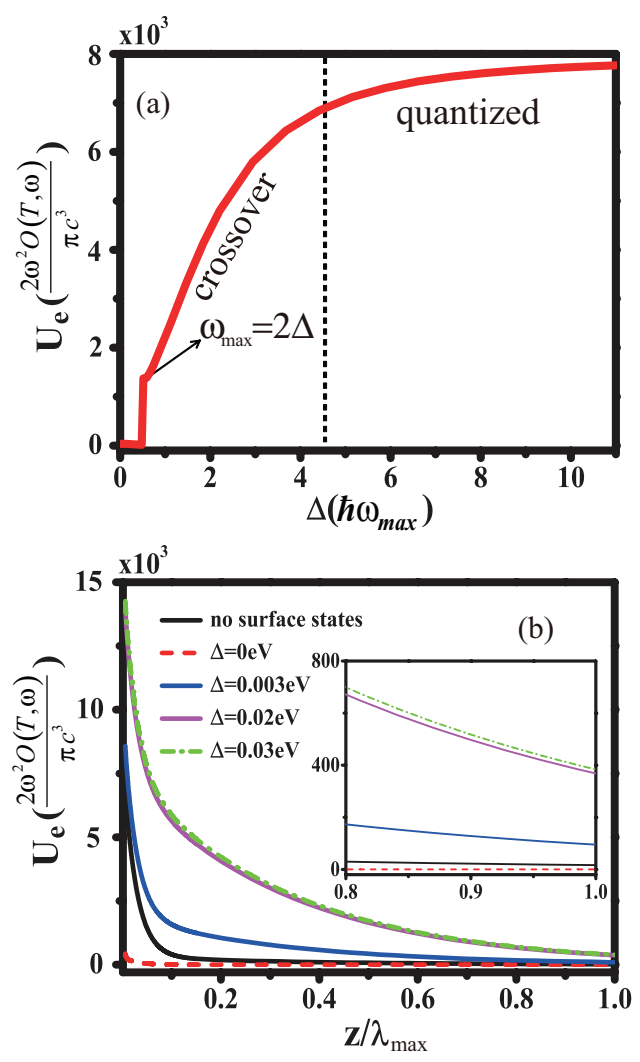

FIG. 5. (Color online) (a) Plot of the thermal radiation energy density $\left(\mathbf{U}_{e}\right.$ versus the surface gap $\Delta$ on the plane $z=\lambda_{\max } / 20$ above the upper TI/vacuum interface. (b) Plot of $\mathbf{U}_{e}$ versus the height $z$ for various surface gaps.
Fig. 5(b), we plot $\mathbf{U}_{e}$ as a function of $z$, which measures the height of the detection plane from the upper TI/vacuum interface. Due to the nonvanishing $\sigma_{x x}^{R}$ and $\sigma_{x y}^{I}$ in the interband transition regime, $\mathbf{U}_{e}$ [red curve in Fig. 5(b)] is even lower than that of a dielectric slab [black curve in Fig. 5(b)]. Remarkably, for a specified case the thickness $h=0.116 \lambda_{\max } / 2$, the gapped surface state can significantly enhance $\mathbf{U}_{e}$ (up to 7500 times of the black-body radiation energy at the given frequency), when the system enters the HIQSHC regime [see Fig. 5(a)].

\section{COMPARISON WITH THE SYSTEMS SUPPORTING SURFACE STATES}

Before we make the conclusions, we would like to compare our results with the systems supporting surface states. ${ }^{1}$ By using the definition of the coherence length (23), we calculate the coherence length of $\mathbf{W}_{x x}$ by probing the radiation field on the plane $z=\lambda_{\max } / 20$ above the SiC surface [see Fig. 2(b) in Ref. 1]. The coherence length $\ell_{x x}$ in their case is about $0.61 \lambda_{\max }$, which is only half of the coherence length $\ell_{x x}$ for the TI slab of the thickness $h=0.116 \lambda_{\max } / 2$ in the HIQSHC regime [see Fig. 3(b)]. We also calculate and compare the coherence lengths of $\mathbf{W}_{y y}$ and $\mathbf{W}_{z z}$ for these two materials. For both of them, the coherence lengths for the TI slab is slightly longer than those for $\mathrm{SiC}$ material. For example, the coherence length of $\mathbf{W}_{y y}$ is $0.32 \lambda_{\max }$ for the TI slab and $0.29 \lambda_{\max }$ for $\mathrm{SiC}$, and the coherence length of $\mathbf{W}_{z z}$ is $0.68 \lambda_{\max }$ for the TI slab and $0.62 \lambda_{\max }$ for $\mathrm{SiC}$.

On the other hand, the radiation energy density on the plane $z=\lambda_{\max } / 20$ above the SiC surface is found to be about 400 times of the black-body radiation energy density at the given frequency. However, due to the photon energy for Ref. 1 is much larger (about 50 times of the photon energy under the consideration in the present study). Therefore, the radiation energy density for $\mathrm{SiC}$ systems is about three times that of the TI slab of the thickness $h=0.116 \lambda_{\max } / 2$ in the HIQSHC regime.

From the comparison, we notice that the low working frequency is the main problem of TI slabs as thermal radiation sources. This big problem is mainly due to the small surface gap (typically in the order of $0.01 \mathrm{eV}$ ). As demonstrated, a higher working frequency would drive the system into the interband transition regime, in which the coupling between the surface states and waveguide modes is generally small and cannot provide much improvement to the coherence of the thermal radiation field.

\section{CONCLUDING REMARKS}

In this work, we first develop a general Green's-function method applied to the slab geometry. Given the advantages of the method, we can handle quite complex conversions between different polarized modes at the interface. By using the method, we study the effects of the surface states on the coherence properties of the thermal radiation field from a TI slab. We find when the surface gap is larger than the photon energy of the radiation field, the strong coupling between the surface states and waveguide modes can change the coherence properties of the thermal radiation field very much. As a specified example we consider the TI slab of a thickness $h=0.116 \lambda_{\max } / 2$ and 
probe the thermal radiation field on the plane $z=\lambda_{\max } / 20$ above the upper TI/vacuum interface. Though the radiation energy density from the TI slab is about $1 / 3$ of that from SiC, surprisingly, the coherence length of $\mathbf{W}_{x x}$ for the TI slab can be remarkably as long as twice that for $\mathrm{SiC}$.

\section{ACKNOWLEDGMENTS}

We would like to thank S. Louie, K.-M. Ho, and Z.-H. Hang for useful comments and suggestions. This work was supported by HKUST2/CRF/11G. B.H. would like to thank A Project Funded by the Priority Academic Program Development of Jiangsu Higher Education Institutions (PAPD) and the National Natural Science Foundation of China (Grant No. 11104198) for support.

\section{APPENDIX A: EXPRESSIONS OF THE TRANSMISSION COEFFICIENTS}

By solving the equation obtained by matching boundary conditions at the TI/vacuum interfaces, we can solve out the radiation field in region III (above the upper TI/vacuum interface). In Eq. (14) of the main text, we expressed the radiation field by transmission coefficients. Considering the expressions are too long, we do not give the explicit form in the main text. Here we would like to provide the detailed expressions, since in Sec. III of the main text we have to use the expression to determine the position of the resonance.
As demonstrated, the transmission coefficients leading to the TM modes in vacuum would have the same denominator:

$$
D_{p}=\left(1+\Theta_{p}\right)^{2}-\left(r_{p}-r_{s} \Theta_{p}\right)^{2} e^{i 2 k_{z, \mathrm{TI}} h},
$$

and the transmission coefficients leading to the TE modes in vacuum have also the same denominator:

$$
D_{s}=\left(1+\Theta_{s}\right)^{2}-\left(r_{s}-r_{p} \Theta_{s}\right)^{2} e^{i 2 k_{z, \mathrm{TI}} h},
$$

where $r_{p}$ and $r_{s}$ have been defined in the main text, $\Theta_{p}$ and $\Theta_{s}$ are given by

$$
\begin{gathered}
\Theta_{p}=\frac{\Upsilon^{2} \beta\left(1-e^{i 2 k_{z, \mathrm{TI}} h}\right)}{\Omega \Lambda\left(1-r_{s}^{2} e^{i 2 k_{z, \mathrm{TI}} h}\right)}, \\
\Theta_{s}=\frac{\Upsilon^{2} \beta\left(1-e^{i 2 k_{z, \mathrm{TI}} h}\right)}{\Omega \Lambda\left(1-r_{p}^{2} e^{i 2 k_{z, \mathrm{TI}} h}\right)} .
\end{gathered}
$$

In the above equations, the coefficients $\Upsilon, \Omega$, and $\Lambda$ are given by

$$
\begin{aligned}
& \Upsilon=-\frac{4 \pi \sigma_{x y}}{c \pi} \sqrt{\frac{\mu}{\varepsilon}}, \\
& \Omega=\sqrt{\frac{\mu}{\varepsilon}}+\beta+\frac{4 \pi k_{z, V} \sigma_{x x}}{\omega} \sqrt{\frac{\mu}{\varepsilon}}, \\
& \Lambda=1+\sqrt{\frac{\mu}{\varepsilon}} \beta+\frac{4 \pi \omega \mu \sigma_{x x}}{c^{2} k_{z, \mathrm{TI}}} .
\end{aligned}
$$

By using these parameters, the transmission coefficients are given by

$$
\begin{aligned}
& T_{p,+; s, g,+}=\frac{\left(-2 \Upsilon \Theta_{p} B_{1} e^{i k_{z, \mathrm{TI}}\left(h-z^{\prime}\right)}-2 \Upsilon B_{1} e^{i k_{z, \mathrm{TI}}\left(h-z^{\prime}\right)}+2 r_{p} \Upsilon B_{2} e^{i k_{z, \mathrm{TI}}\left(3 h-z^{\prime}\right)}-2 r_{s} \Upsilon \Theta_{p} B_{2} e^{i k_{z, \mathrm{TI}}\left(3 h-z^{\prime}\right)}\right)}{e^{i k_{z, V} h} D_{p}}, \\
& T_{p,+; s, g,-}=\frac{\left(2 \Upsilon \Theta_{p} B_{2} e^{i k_{z, \mathrm{TI}}\left(h+z^{\prime}\right)}+2 \Upsilon B_{2} e^{i k_{z, \mathrm{TI}}\left(h+z^{\prime}\right)}-2 r_{p} \Upsilon B_{1} e^{i k_{z, \mathrm{TI}}\left(h+z^{\prime}\right)}+2 r_{s} \Upsilon \Theta_{p} B_{1} e^{i k_{z, \mathrm{TI}}\left(h+z^{\prime}\right)}\right)}{e^{i k_{z, V} h} D_{p}}, \\
& T_{p,+; p, g,+}=\frac{\left(\frac{2 \Theta_{p}}{\Omega} e^{i k_{z, \mathrm{TI}}\left(h-z^{\prime}\right)}+\frac{2}{\Omega} e^{i k_{z, \mathrm{TI}}\left(h-z^{\prime}\right)}\right)}{e^{i k_{z, V} h} D_{p}}, \\
& T_{p,+; p, g,-}=\frac{\left(\frac{2 r_{p}}{\Omega} e^{i k_{z, \mathrm{TI}}\left(h+z^{\prime}\right)}-\frac{2 r_{s} \Theta_{p}}{\Omega} e^{i k_{z, \mathrm{TI}}\left(h+z^{\prime}\right)}\right)}{e^{i k_{z, V} h} D_{p}}, \\
& T_{s,+; s, g,+}=\frac{\left(\frac{2 \Theta_{s}}{\Lambda} e^{i k_{z, \mathrm{TI}}\left(h-z^{\prime}\right)}+\frac{2}{\Lambda} e^{i k_{z, \mathrm{TI}}\left(h-z^{\prime}\right)}\right)}{e^{i k_{z, V} h} D_{s}}, \\
& T_{s,+; s, g,-}=\frac{\left(\frac{2 r_{s}}{\Lambda} e^{i k_{z, \mathrm{TI}}\left(h+z^{\prime}\right)}-\frac{2 r_{p} \Theta_{s}}{\Lambda} e^{i k_{z, \mathrm{TI}}\left(h+z^{\prime}\right)}\right)}{e^{i k_{z, V} h} D_{s}}, \\
& T_{s,+; p, g,+}=\frac{\left(2 \Upsilon \Theta_{s} B_{3} e^{i k_{z, \mathrm{TI}}\left(h-z^{\prime}\right)}+2 \Upsilon B_{3} e^{i k_{z, \mathrm{TI}}\left(h-z^{\prime}\right)}-2 r_{p} \Upsilon \Theta_{s} B_{4} e^{i k_{z, \mathrm{TI}}\left(3 h-z^{\prime}\right)}+2 r_{s} \Upsilon B_{4} e^{i k_{z, \mathrm{TI}}\left(3 h-z^{\prime}\right)}\right)}{e^{i k_{z, V} h} D_{s}}, \\
& T_{s,+; p, g,-}=\frac{\left(2 \Upsilon \Theta_{s} B_{4} e^{i k_{z, \mathrm{TI}}\left(h+z^{\prime}\right)}+2 \Upsilon B_{4} e^{i k_{z, \mathrm{TI}}\left(h+z^{\prime}\right)}-2 r_{p} \Upsilon \Theta_{s} B_{3} e^{i k_{z, \mathrm{TI}}\left(h+z^{\prime}\right)}+2 r_{s} \Upsilon B_{3} e^{i k_{z, \mathrm{TI}}\left(h+z^{\prime}\right)}\right)}{e^{i k_{z, V} h} D_{s}},
\end{aligned}
$$

where the four parameters $B_{1}, B_{2}, B_{3}$, and $B_{4}$ are given by

$$
\begin{aligned}
& B_{1}=\frac{1-r_{s} e^{i 2 k_{z, \mathrm{TI}} h}}{\Omega \Lambda\left(1-e^{i 2 k_{z, \mathrm{TI}} h} r_{s}^{2}\right)}, \\
& B_{2}=\frac{1-r_{s}}{\Omega \Lambda\left(1-e^{i 2 k_{z, \mathrm{TI}} h} r_{s}^{2}\right)},
\end{aligned}
$$

$$
\begin{aligned}
& B_{3}=\frac{\beta\left(1+r_{p} e^{i 2 k_{z, \mathrm{TI}} h}\right)}{\Omega \Lambda\left(1-e^{i 2 k_{z, \mathrm{TI}} h} r_{p}^{2}\right)}, \\
& B_{4}=\frac{\beta\left(1+r_{p}\right)}{\Omega \Lambda\left(1-e^{i 2 k_{z, \mathrm{TI}} h} r_{s}^{2}\right)} .
\end{aligned}
$$



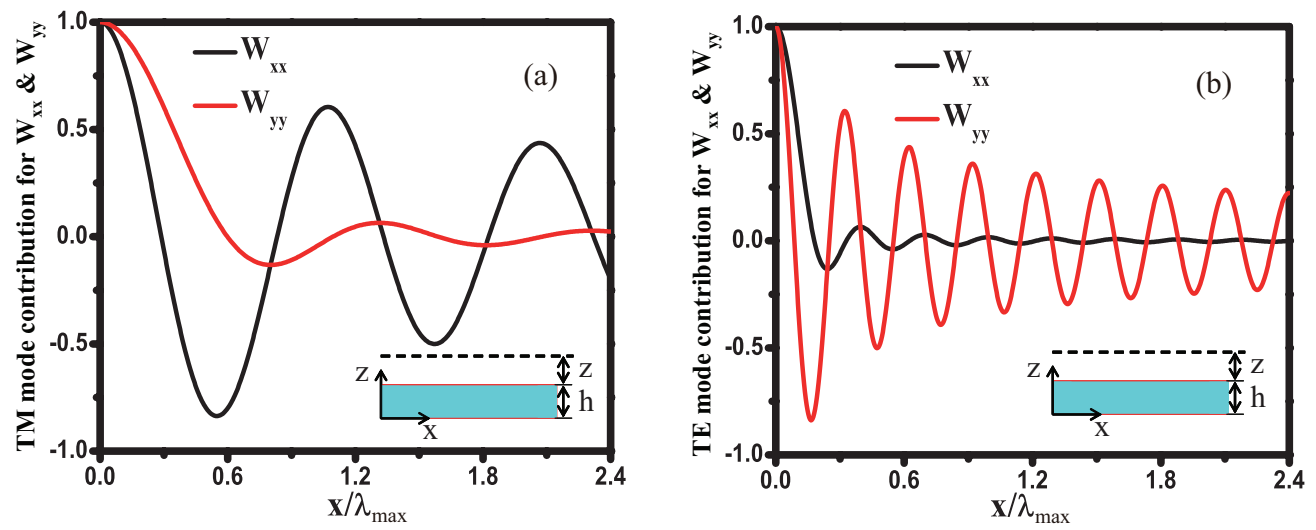

FIG. 6. (Color online) (a) $\mathbf{W}_{x x}\left(\left|\vec{R}_{1}-\vec{R}_{2}\right|\right)$ and $\mathbf{W}_{y y}\left(\left|\vec{R}_{1}-\vec{R}_{2}\right|\right)$ versus $x=\left|\vec{R}_{1}-\vec{R}_{2}\right|$ for the TM radiation mode from a dielectric slab; (b) $\mathbf{W}_{x x}\left(\left|\vec{R}_{1}-\vec{R}_{2}\right|\right)$ and $\mathbf{W}_{y y}\left(\left|\vec{R}_{1}-\vec{R}_{2}\right|\right)$ versus $x=\left|\vec{R}_{1}-\vec{R}_{2}\right|$ for the TE radiation mode from a dielectric slab.

\section{APPENDIX B: SPATIAL COHERENCE OF TE AND TM MODES}

We consider a dielectric slab of no surface state. To explicitly demonstrate the difference between the TM and TE radiation modes, we plot the spatial coherence $\left(\mathbf{W}_{x x}\right.$ and $\left.\mathbf{W}_{y y}\right)$ of the TM polarized thermal radiation field in Fig. 6(a), and that of the TE polarized thermal radiation field in Fig. 6(b). The calculation is performed by probing the thermal radiation field on the plane $z=\lambda_{\max } / 20$ above the upper TI/vacuum interface at the maximal radiation frequency of $T=6 \mathrm{~K}$ $\left(\hbar \omega_{\max } \approx 0.0026 \mathrm{eV}\right)$. The thickness of the TI is set to be $h=0.116 \lambda_{\max } / 2$ (the same with the main text). From the dispersion relations of the waveguide modes, we note that for the same frequency, the TM mode always bears a smaller tangential wave vector. It explains the fact that the periodicity of the spatial coherence of TM mode [Fig. 6(a)] is larger than that of TE mode [Fig. 6(b)]. Since we are interested in the spatial coherence along a straight line in the $x$ direction connecting two observation points, the waveguide modes with larger $x$-component wave vector should have a larger contribution. A larger $x$-component wave vector would result in larger $y$-component electric field for TE modes but larger $x$-component electric field for TM modes. As a consequence, one observes better coherence for the $y$ component of TE mode and the $x$ component of TM mode.

\footnotetext{
*phwen@ust.hk

${ }^{1}$ R. Carminati and J.-J. Greffet, Phys. Rev. Lett. 82, 1660 (1999).

${ }^{2}$ C. Henkel, K. Joulain, R. Carminati, and J.-J. Greffet, Opt. Commun. 186, 57 (2000).

${ }^{3}$ J.-J. Greffet, R. Carminati, K. Joulain, J.-P. Mulet, S. Mainguy, and Y. Chen, Nature (London) 416, 61 (2002).

${ }^{4}$ A. I. Volokitin and B. N. J. Persson, Rev. Mod. Phys. 79, 1291 (2007).

${ }^{5}$ I. A. Dorofeyev and E. A. Vinogradov, Phys. Rep. 504, 75 (2011).

${ }^{6}$ W. T. Lau, J. T. Shen, G. Veronis, and S. H. Fan, Phys. Rev. E 76, 016601 (2007).

${ }^{7}$ S. E. Han, Phys. Rev. B 80, 155108 (2009).

${ }^{8}$ L. Mandel and E. Wolf, Optical Coherence and Quantum Optics (Cambridge University Press, Cambridge, England, 1995).

${ }^{9}$ Y. De Wilde, F. Formanek, R. Carminati, B. Gralak, P. A. Lemoine, K. Joulain, J. P. Mulet, Y. Chen, and J. J. Greffet, Nature (London) 444, 740 (2006)

${ }^{10}$ S. John and R. Wang, Rev. Rev. A 78, 043809 (2008).

${ }^{11}$ P. Nagpal, S. E. Han, A. Stein, and D. J. Norris, Nano Lett. 8, 3238 (2008).

${ }^{12}$ I. Celanovic, D. Perreault, and J. Kassakian, Phys. Rev. B 72, 075127 (2005).

${ }^{13}$ C. M. Cornelius and J. P. Dowling, Phys. Rev. A 59, 4736 (1999).
}

${ }^{14}$ S. Y. Lin, J. Moreno, and J. G. Fleming, Appl. Phys. Lett. 83, 380 (2003).

${ }^{15}$ C. Luo, A. Narayanaswamy, G. Chen, and J. D. Joannopoulos, Phys. Rev. Lett. 93, 213905 (2004).

${ }^{16}$ S. Shen, A. Narayanaswamy, and G. Chen, Nano. Lett. 9, 2909 (2009).

${ }^{17}$ E. Rousseau, A. Siria, G. Jourdan, S. Volz, F. Comin, J. Chevrier, and J.-J. Greffet, Nat. Photon. 3, 514 (2009).

${ }^{18}$ P. J. van Zwol, L. Ranno, and J. Chevrier, Phys. Rev. Lett. 108, 234301 (2012).

${ }^{19}$ C. L. Kane and E. J. Mele, Phys. Rev. Lett. 95, 146802 (2005); 95, 226801 (2005).

${ }^{20}$ B. A. Bernevig, T. L. Hughes, and S.-C. Zhang, Science 314, 1757 (2006).

${ }^{21}$ L. Fu, C. L. Kane, and E. J. Mele, Phys. Rev. Lett. 98, 106803 (2007).

${ }^{22}$ J. E. Moore and L. Balents, Phys. Rev. B 75, 121306(R) (2007).

${ }^{23}$ X.-L. Qi, T. L. Hughes, and S.-C. Zhang, Phys. Rev. B 78, 195424 (2008).

${ }^{24}$ L. Fu and C. L. Kane, Phys. Rev. B 76, 045302 (2007).

${ }^{25}$ X.-L. Qi, R. Li, J. Zang, and S.-C. Zhang, Science 323, 1184 (2009). 
${ }^{26}$ A. G. Grushin and A. Cortijo, Phys. Rev. Lett. 106, 020403 (2011).

${ }^{27}$ W. K. Tse and A. H. MacDonald, Phys. Rev. Lett. 105, 057401 (2010).

${ }^{28}$ J. E. Sipe, J. Opt. Soc. Am. B 4, 481 (1987).

${ }^{29}$ L. Fu, Phys. Rev. Lett. 103, 266801 (2009).

${ }^{30}$ C. X. Liu, X. L. Qi, H. J. Zhang, X. Dai, Z. Fang, and S. C. Zhang, Phys. Rev. B 82, 045122 (2010).

${ }^{31}$ G. Mahan, Many-particle Physics (Kluwer Academic, Dordrecht, 1990).
${ }^{32} \mathrm{H}$. Bruus and K. Flensberg, Many-body Quantum Theory in Condensed Matter Physics: An Introduction (Oxford University Press, New York, 2004).

${ }^{33}$ N. A. Sinitsyn, J. E. Hill, H. Min, J. Sinova, and A. H. MacDonald, Phys. Rev. Lett. 97, 106804 (2006).

${ }^{34}$ L. A. Falkovsky and S. S. Pershoguba, Phys. Rev. B 76, 153410 (2007).

${ }^{35}$ L. D. Landau, E. M. Lifshitz, and L. P. Pitaevskii, Statistics Mechanics (Pergamon, Oxford, 1980). 